\title{
Comparison of Selected Physical and Performance Characteristics in University-Level Male Basketball, Football and Volleyball Players
}

\author{
Meltem Koç ${ }^{1 \mathrm{D}}$, Özge İpek DONGAZ ${ }^{2}$, Banu BAYAR ${ }^{3}$ and Kılıçhan BAYAR ${ }^{(\mathrm{DD}}$ \\ 1,2,3,4 Muğla Sitkı Koçman University, Faculty of Health Sciences, Department of Physiotherapy and Rehabilitation, Muğla, \\ Turkey \\ *Corresponding author: kcmeltemm@gmail.com
}

\begin{abstract}
Abstrac
The purpose of present study was to compare the selected physical and performance variables of university-level basketball, football and volleyball players. The present study was conducted by 23 students studying in the faculty of health sciences and playing in their school's Football $(n=7)$, Volleyball $(n=8)$, Basketball $(n=8)$ teams and the age ranged from 18-23 years. Several physical and physiological characteristics of the students were evaluated. These characteristics are weight, height, BKI, flexibility, isometric muscle strength, muscular endurance, aerobic and anaerobic performance. While comparing between sports disciplines, one-way analysis of variance (ANOVA) was used for data with normal distribution, and Kruskal Wallis test was used for non-distributed data. The results of the study indicate that there was significant difference among Basketball, Volleyball and Football in relation to VO2 Max, muscle strength, anaerobic power. The aerobic performance test was significantly better in volleyball $(\mathrm{p} \leq 0.05)$. However, there was no statistically significant difference in the results of physical properties, muscle endurance and flexibility among teams $(\mathrm{p}>0.05)$. These results will provide useful information for university level athletes to be selected according to sports-specific physical fitness criteria and that they should receive training by team coaches accordingly.
\end{abstract}

\section{Keywords}

Physical Fitness, Basketball, Volleyball, Football

\section{INTRODUCTION}

Success in sports is made possible by the complex interaction of many factors such as motivation level, psychosocial status, physical and physiological characteristics of players in both individual and team sports. However, physical fitness is an important component when considering the nature of the sport that is considered to be chosen and also the maintenance of success in that particular sports type. In team sports, a single anthropometric feature or fitness profile is generally not expected to be strong.
The reason for this is that such sports are competitive and it requires many fitness profiles in order to be good together as a team, furthermore their complex relationships with each other are also very significant. For example, a volleyball player is expected to have significant strength, flexibility, speed, agility and endurance. However, some parameters may have a more profound effect on success in sports when compared with others. For example, the vertical jump is more important for basketball and volleyball players, rather than football players. For this reason, reference fitness profiles have been created in many studies with 
elite athletes (Di Salvo et al., 2007; Peña, MorenoDoutres, Coma, Cook, \& Buscà, 2018). In amateur teams, school teams or recreational sports, these fitness features, in general, are not considered. However, the fact that some athletes, with an inappropriate fitness profile, take place in a sport discipline, will not only decrease the success in sports but also may cause serious injuries. However, although the physical fitness characteristics of elite players are known, the data on these features of university level players are limited. Therefore, in the present study, it was aimed to analyze and compare some anthropometric and physical properties of football, volleyball and basketball teams created for a sports competition between faculties in a university. The results of the present study with university-level players will provide useful information to other professionals interested in sports, especially team coaches.

\section{MATERIALS AND METHODS}

The present study was conducted as crosssectional descriptive study design. The local University Ethics Committee approved the study protocol design (ethic code: 80/81), which respected the principles of the Declaration of Helsinki (1975 and further updates). All the individuals signed an informed consent form. The present study included 23 male students playing on the faculty school team and agreeing to participate in the study voluntarily.

Inclusion criteria were as follows:

- Students who have played on the faculty team for at least two years

- Students who regularly participate in training sessions twice a week and to play in the team in inter-university competitions at the end of the year.

Exclusion criteria were as follows:

- Students who smoked, had chronic disease and had any surgery in the last 5 years.

The students participating in the study were divided into 3 groups as football $(n=7)$, basketball $(n=8)$ and volleyball $(n=8)$ according to the team they played. Some different selected physical variables (height, body weight) and performance characteristics (flexibility, muscle strength, muscular endurance, aerobic and anaerobic performance) were evaluated. This approach allowed comparisons between teams to define a fitness profile in each of them. All evaluations were made by evaluators (M.K and O.I) who did not know which team the student was playing. The subjects were allowed to rest for $5 \mathrm{~min}$ between tests.

\section{Procedures}

The following describes how physical fitness components are assessed.

\section{Height, Weight and BMI}

Height was measured to the nearest $0.1 \mathrm{~cm}$ using a wall-mounted stadiometer, and weight was measured to the nearest $0.1 \mathrm{~kg}$ using a digital scale after all outer clothing (only formal team clothes) and shoes were removed. In addition, Body mass index (BMI) calculation was performed for weight body $(\mathrm{kg})$ / height $(\mathrm{m})^{2}$ formula (Deurenberg, Weststrate, \& Seidell, 1991).

\section{Flexibility}

A sit and reach test was performed to determine the lumbar region and hamstring flexibility of the individuals. A standard sit and reach test was performed using an sit and reach box with a height of $30.6 \mathrm{~cm}$. The box was set on an examining table (height $74 \mathrm{~cm}$ ) where the sit and reach test was performed. The subjects sat with their feet approximately hip-wide against the testing box. They kept their knees extended and placed the right hand over the left, and slowly reached forward as far as they could by sliding their hands along the measuring board. Reaches short of the toes were recorded as negative forward reach scores and reaches beyond the toes were recorded as positive forward reach scores. The test was repeated three times and the best value was recorded (López-Miñarro, Andújar, \& RodrñguezGarcña, 2009).

\section{Anaerobic Power}

Participants' anaerobic power was assessed with vertical jump (VJ) test. First, when the feet were adjacent and the body was in a vertical position, the double-arm was extended up to mark the last point where the fingertips contacted. Then the participant tried to contact the board by splashing all the power up with the double foot (This should not take a step during the upward 
bounce, but it may bend the knees). Secondly, the place where the board was contacted was marked. The distance between two points was measured. This process was repeated three times and the best value was recorded. Anaerobic power was calculated using the Lewis formula using vertical jump distance and body weight.

Average Power $=($ square root of 4.9) $\mathrm{x}$ body mass $(\mathrm{kg}) \times($ square root of jump distance $(\mathrm{m})$ )

\section{Aerobic Performance}

Aerobic performance was tested by the Cooper performance test. Individuals were told to run for 12 minutes on the track at the speed they wanted on the track, or if they could not keep running, they would walk. For the test, subjects were encouraged to run/jog the entire distance but were told they could walk, if necessary. The total distance (in meters) taken at the end of 12 minutes was calculated by multiplying the total number of tours with 400 meters (the distance traveled in the last non-completed tour was added as a meter). The distance unit was converted from metro to $\mathrm{km}$. The following formula was used to estimate $\mathrm{VO}_{2}$ max (Meredith \& Welk, 2003).

Estimate $\mathrm{VO}_{2}$ maximum $\left(\mathrm{ml} \cdot \mathrm{kg}^{-1} \cdot \mathrm{min}^{-1}\right)=$ (22.351 x distance covered in kilometers) - 11.288

\section{Muscle Strength}

The Fei Lafayette Manuel Muscle Tester electronic dynamometer was used to determine the isometric muscle strength of individuals (Lafayette Instrument Company, Lafayette, Ind., USA). Muscle strength of 3 gross muscle groups (shoulder flexor, hyper extensor and abductor) in the upper extremity and 4 gross muscle groups in the lower extremity (knee extensor and flexor, hip extensor and flexor) were evaluated by the same researcher (O.I). All the tests performed to evaluate the maximum isometric muscle strength were repeated twice, giving a rest interval of one minute. The digital score on the dynamometer for the peak values was recorded for each trial and the best score was recorded in kilograms (Andrews, Thomas, \& Bohannon, 1996; Fosang \& Baker, 2006).

\section{Muscle endurance}

The push-ups test and the sit-ups test were used to assess muscle endurance. Between the two tests, individuals were given a resting time of 5 minutes. Standard Push-up test used for assessing the endurance of the arm, shoulder extensor muscle group and trunk stabilizer muscles. When executing a push-up an individual start in the "up" position with the arms straight, lowers the body to the "down" position, and then raises it to the up position. Throughout the execution of a push-up, the body is supposed to be kept straight. When the push-up is used as a test, failure to correctly assume the down position or up position, or keep the body straight, results in a push-up not being counted. The researchers recorded the number of correct push-ups in the 1-minute push-up test. Participants were encouraged to give maximal effort during the test. The detailed test procedure of the push-up test was carried out as stated by Baumgartner et al (Baumgartner, Oh, Chung, \& Hales, 2002).

Standard Sit-ups test was used for assessing the endurance of abdominal muscles and hip flexors. The sit-up protocol required the participant to perform as many bent knee sit-ups as possible in 1 minute. Participants laid in a supine position with the knees bent and feet flat on a mat. The hands were placed on the side of the head with fingers over the ears. The participants elevated the trunk until the elbows made contact with the legs. They reversed directions until the shoulder blades touched the mat. The feet were secured by the test examiner who counted the sit-ups during the 1minute test. Participants were encouraged to give maximal effort during the test (Jackson et al., 1998).

\section{Statistical Analysis}

Statistical analysis of the study was done using the SPSS 22.00 Windows package program. The data were expressed as mean \pm standard deviation $(X \pm S D)$. Before the use of any parametric or nonparametric test, each log normality analysis was performed with Kolmogorov-Smirnov. In comparison of physical fitness components, One-way ANOVA was used for the data with normal distribution and KruskalWallis test was used for data with non-distribution data. When significant differences were found, we proceeded to compare between groups with a Tukey post hoc analysis in the case of the ANOVA, and Games-Howell post hoc analysis for Kruskal-Wallis. The significance level for the tests was established at $p \leq 0.05$. 


\section{RESULTS}

Anthropometrically, there was no statistically significant difference between the players of the three teams according to the oneway ANOVA. The average age, height, body weight and BMI of the teams are given in Table 1.

Table 1: Physical features of the subjects participating in the study

\begin{tabular}{llll}
\hline & $\begin{array}{l}\text { Male Basketball } \\
\text { Mean } \pm \text { sd }\end{array}$ & $\begin{array}{l}\text { Male Volleyball } \\
\text { Mean } \pm \text { sd }\end{array}$ & $\begin{array}{l}\text { Male Football } \\
\text { Mean } \pm \text { sd }\end{array}$ \\
\hline Age $($ year $)$ & $20.62 \pm 1.68$ & $20.12 \pm 0.64$ & $21.42 \pm 0.78$ \\
\hline Height $(\mathrm{m})$ & $1.82 \pm 0.05$ & $1.78 \pm 0.44$ & $1.77 \pm 0.09$ \\
\hline Weight $(\mathrm{kg})$ & $74.37 \pm 12.87$ & $73.62 \pm 7.34$ & $75.42 \pm 9.6$ \\
\hline BMI $\left(\mathrm{kg} / \mathrm{m}^{2}\right)$ & $22.24 \pm 3.29$ & $23.15 \pm 1.94$ & $24.02 \pm 2.27$ \\
\hline
\end{tabular}

BMI: Body Mass Index

There was no statistically significant difference between the teams in sit-and reach test and muscle endurance tests. VJ tests indicated significant differences between teams $(p<0.05)$. Basketball players showed better performances when compared to volleyball and football players in the VJ test. Similarly, Cooper tests indicated significant differences between teams $(\mathrm{p}<0.05)$. Volleyball players had a higher cooper distance average than other sports teams (Table 2).

Table 2: Performance test results of the subjects participating in the study

\begin{tabular}{lccc}
\hline & $\begin{array}{c}\text { Male Basketball } \\
\mathrm{X} \pm \mathrm{SD}\end{array}$ & $\begin{array}{c}\text { Male Volleyball } \\
\mathrm{X} \pm \mathrm{SD}\end{array}$ & $\begin{array}{c}\text { Male Football } \\
\mathrm{X} \pm \mathrm{SD}\end{array}$ \\
\hline Sit and Reach Test $(\mathrm{cm})$ & $-5 \pm 12.27$ & $1.5 \pm 10.83$ & $0.85 \pm 13.59$ \\
\hline Vertical Jump Test & & & \\
$\quad$ Power $(\mathrm{kg} \cdot \mathrm{m} / \mathrm{sn}) *^{\mathrm{a}, \mathrm{b}}$ & $111.13 \pm 21.10$ & $118.25 \pm 11.49$ & $120.57 \pm 15.91$ \\
Distance $(\mathrm{cm})^{\mathrm{a}, \mathrm{b}}$ & $53.62 \pm 5.26$ & $47.87 \pm 4.48$ & $45.1 \pm 2.34$ \\
\hline Cooper Test & & & \\
MaxVO $_{2}\left(\mathrm{ml} \cdot \mathrm{kg}^{-1} \cdot \mathrm{min}^{-1}\right)^{*{ }^{\mathrm{a}}}$ & $31.17 \pm 5.85$ & $42.63 \pm 6.59$ & $25.77 \pm 20.73$ \\
Distance $(\mathrm{km})^{\text {Muscular Endurance }}$ & $1.90 \pm 0.2$ & $2.41 \pm 0.2$ & $1.42 \pm 0.9$ \\
$\quad$ Sit-ups & $30.1 \pm 4.29$ & $43.85 \pm 17.28$ & $35.85 \pm 11.45$ \\
Push-ups & $24.6 \pm 14.37$ & $24.75 \pm 14.45$ & $25.85 \pm 12.96$ \\
\hline${ }^{*} \mathrm{p}<0.05,{ }^{\mathrm{a}}$ Basketball vs. volleyball, ${ }^{\mathrm{b}}$ Basketball vs. football, ${ }^{\mathrm{c}}$ football vs. volleyball &
\end{tabular}

In the isometric muscle strength tests, when the right-side muscle strengths of the teams were compared, there was a significant difference between the teams except shoulder extension, abduction and hip flexion. When the left-side muscle strengths were compared, there was a significant difference between the teams except for shoulder abduction. The values obtained from the test and the differences between teams are presented in Table 3. 
Table 3: Values obtained from isometric muscle strength test (expressed in $\mathbf{N ~ m / k}$ )

\begin{tabular}{|c|c|c|c|c|}
\hline & & $\begin{array}{c}\text { Basketball }(\mathrm{n}=8) \\
\mathrm{X} \pm \mathrm{SD}\end{array}$ & $\begin{array}{c}\text { Volleyball }(\mathrm{n}=7) \\
\mathrm{X} \pm \mathrm{SD}\end{array}$ & $\begin{array}{c}\text { Football }(n=8) \\
\quad X \pm S D\end{array}$ \\
\hline \multirow[t]{2}{*}{ Arm Flexor } & Right $^{* b, c}$ & $16.87 \pm 1.19$ & $21.02 \pm 2.32$ & $15.31 \pm 1.94$ \\
\hline & Left $*$ b,c & $16.07 \pm 1.41$ & $22.22 \pm 1.81$ & $14.76 \pm 2.27$ \\
\hline \multirow[t]{2}{*}{ Arm Abductor } & Right $^{* b, c}$ & $15.93 \pm 1.79$ & $21.54 \pm 2.16$ & $16.63 \pm 3.90$ \\
\hline & Left $*$ b & $15.33 \pm 1.13$ & $20.45 \pm 2.13$ & $16.46 \pm 4.35$ \\
\hline \multirow[t]{2}{*}{ Arm Extansor } & Right & $11.67 \pm 1.41$ & $12.78 \pm 2.86$ & $12.57 \pm 2.60$ \\
\hline & Left & $10.96 \pm 0.61$ & $13.20 \pm 1.96$ & $11.20 \pm 3.46$ \\
\hline \multirow[t]{2}{*}{ Knee Flexor } & Right & $18.03 \pm 1.99$ & $16.12 \pm 7.53$ & $16.78 \pm 2.81$ \\
\hline & Left & $17.23 \pm 2.22$ & $19.25 \pm 4.23$ & $16.01 \pm 2.53$ \\
\hline \multirow[t]{2}{*}{ Knee Extansor } & Right & $16.76 \pm 1.69$ & $15.27 \pm 7.40$ & $15.45 \pm 3.16$ \\
\hline & Left & $15.78 \pm 1.92$ & $17.78 \pm 1.67$ & $15.17 \pm 4.36$ \\
\hline \multirow[t]{2}{*}{ Hip Flexor } & Right $^{* b}$ & $18.38 \pm 0.66$ & $21.81 \pm 2.20$ & $21.08 \pm 3.85$ \\
\hline & Left $*$ b & $18.45 \pm 1.25$ & $21.54 \pm 1.40$ & $19.81 \pm 3.42$ \\
\hline \multirow[t]{2}{*}{ Hip Extansor } & Right $^{* a, c}$ & $18.87 \pm 8.45$ & $22.02 \pm 2.32$ & $16.88 \pm 2.62$ \\
\hline & Left $*^{\mathrm{a}, \mathrm{c}}$ & $21.78 \pm 3.51$ & $21.27 \pm 2.72$ & $16.31 \pm 2.13$ \\
\hline
\end{tabular}

${ }^{*} p<0.05,{ }^{a}$ Basketball vs. volleyball, ${ }^{b}$ Basketball vs. football, ${ }^{c}$ football vs. volleyball.

\section{DISCUSSION}

In the present study, some physical and performance-related features of university-level male football, basketball and volleyball players were compared. The findings of the study confirmed that there was a significant difference obtained on anaerobic power, aerobic performance and isometric muscular strength. On the other hand, there was no significant difference obtained on physical properties, flexibility and muscular endurance. In the current section, the results of the present study have been compared with other studies researching similar features in universitylevel male football, volleyball and basketball teams.

In this study, there was no difference between the teams in terms of physical characteristics such as height, weight and BMI. However, it is a well-known fact that height is an important determinant for basketball players. Teams that include tall players in basketball have a much significant advantage over other teams, especially in the under-ground fight (Torres-Unda et al., 2013). Unlike performance, especially in adulthood, anthropometric values such as height is not possible to be changed with training. Therefore, this property needs to be taken into consideration during the selection process of the athletes for the basketball team.

Flexibility is one of the most important elements in order to prevent injuries. In the present study, the flexibility value of all teams was very low and there was no significant difference between the teams. Similarly, there was no significant difference in the sit and reach test in Rajveer Singh's study with 30 male universitylevel football and volleyball players (R. Singh, 2019).In present study, the highest VJ distance was found in basketball players. The highest anaerobic power in the VJ test was found in the volleyball players in the study of Marangoz et al. [wrestling $(n=15)$, gymnastics (15), football (15), handball (15), volleyball (15) and basketball $(n=15)]$ with 90 male university-level Turkish athletes from different disciplines (Marangoz \& Baştürk, 2018). Similarly, in a comparison study with the university-level male volleyball $(\mathrm{n}=150)$ and basketball $(\mathrm{n}=150)$ teams, Anita Singh conducted that the VJ test is much higher in volleyball (A. Singh, 2017).

In this study, Max $\mathrm{VO}_{2}$ of volleyball players was quite high compared to the other teams. In a study conducted by Mishra et al., 59 universitylevel males players with age range 20-25, with variables taken from basketball, football and 
volleyball players, it was found that the highest Max $\mathrm{VO}_{2}$ was within the football players (Mishra, Pandey, \& Chaubey, 2015). Bag et al., in a study with university-level male volleyball $(\mathrm{n}=15)$ and football $(\mathrm{n}=15)$ players found that the cardiovascular endurance of football players (1.82 min.) was higher than in volleyball players (Bag, Borman, Das, \& Chawdhury, 2015).

Muscle strength and endurance are an important component for success in team sports when combined with technical skills. In present study, when the sit-up and push-up tests were applied, in terms of muscle endurance there was no significant difference between the teams. In terms of muscle strength, a significant difference in shoulder and hip muscles was observed, however, there was no significant difference observed in knee muscles. Again, in a study conducted by Bag et al., the muscle endurance of football players (39.86 times sit ups) was higher than volleyball players (38.73 times sit ups), and the muscle strength of the football players (5.33 times/min. pull ups) was higher than volleyball players (4.87 times/min. pull ups) (Bag et al., 2015). In Rajveer Singh's study with 30 male university-level football and volleyball players, the football players were better in muscle strength and endurance tests (R. Singh, 2019). Furthermore, a study conducted by Nandalal Singh et al. with 80 players in different sports (20 for each game i.e., football, hockey, basketball and volleyball) found that the highest muscle endurance belonged to football players (T. N. Singh \& Kaur, 2019).

Above, the results of physical characteristics and performance-related features in other Turkish and international players are compared with the results of our study. Results differ from each other. The reason for this may be that in our study, the students were not selected according to the teamspecific physical fitness criteria or their training was insufficient. However, in a sports discipline, basic physical and performance criteria should be provided, otherwise it prevents success, motivation and may cause injuries. In this sense, the results of the present study will provide useful information to team coaches and sports physiotherapists.

\section{REFERENCES}

Andrews, A. W., Thomas, M. W., \& Bohannon, R. W. (1996). Normative values for isometric muscle force measurements obtained with hand-held dynamometers. Physical therapy, 76(3), 248-259.

Bag, A., Borman, A. S., Das, S., \& Chawdhury, B. (2015). Comparative Study on Physical Fitness of Volleyball and Football Players in University Level.

Baumgartner, T. A., Oh, S., Chung, H., \& Hales, D. (2002). Objectivity, reliability, and validity for a revised push-up test protocol. Measurement in Physical Education and Exercise Science, 6(4), 225-242.

Deurenberg, P., Weststrate, J. A., \& Seidell, J. C. (1991). Body mass index as a measure of body fatness: age-and sex-specific prediction formulas. British journal of nutrition, 65(2), 105-114.

Di Salvo, V., Baron, R., Tschan, H., Montero, F. C., Bachl, N., \& Pigozzi, F. (2007). Performance characteristics according to playing position in elite soccer. International journal of sports medicine, 28(03), 222-227.

Fosang, A., \& Baker, R. (2006). A method for comparing manual muscle strength measurements with joint moments during walking. Gait \& Posture, 24(4), 406-411.

Jackson, A. W., Morrow Jr, J. R., Brill, P. A., Kohl III, H. W., Gordon, N. F., \& Blair, S. N. (1998). Relations of sit-up and sit-and-reach tests to low back pain in adults. Journal of Orthopaedic \& Sports Physical Therapy, 27(1), 22-26.

López-Miñarro, P. A., Andújar, P. S. d. B., \& Rodrñguez-Garcña, P. L. (2009). A comparison of the sit-and-reach test and the back-saver sit-and-reach test in university students. Journal of sports science \& medicine, 8(1), 116-122. Retrieved from

Marangoz, İ., \& Baştürk, D. (2018). The Relationship Among Somatotype Structures, Leg Volume, Leg Mass, Anaerobic Strength and Flexibility of Elite Male Athletes in Different Branches. Journal of Education and Training Studies, 6(7), 130-137.

Meredith, M. D., \& Welk, G. (2003). Fitnessgram: Test administration manual: Human Kinetics Publishers.

Mishra, M. K., Pandey, A. K., \& Chaubey, D. (2015). A comparative study of VO2 max among the basketball, football, volleyball and hockey male players. International Journal of Appllied Research, 1(11), 245247. 
Peña, J., Moreno-Doutres, D., Coma, J., Cook, M., \& Buscà, B. (2018). Anthropometric and fitness profile of high-level basketball, handball and volleyball players. Revista Andaluza de Medicina del Deporte, 11(1), 30-35.

Singh, A. (2017). Comparative study of agility, reaction time, strength and flexibility measures of volleyball and basketball players. International Journal of Sport, Exercise and Health Research. 2, 270-272.

Singh, R. (2019). Motor fitness variables among inter university football and volleyball players: a comparative study. International Journal of Physiology, Nutrition and Physical Education, 4(1): 1961-1964

Singh, T. N., \& Kaur, H. (2019). Muscular strength and muscular endurance among national level male players. Human Movement and Sports Sciences, 4(1): 05-07

Torres-Unda, J., Zarrazquin, I., Gil, J., Ruiz, F., Irazusta, A., Kortajarena, M., Irazusta, J. (2013). Anthropometric, physiological and maturational characteristics in selected elite and non-elite male adolescent basketball players. Journal of sports sciences, 31(2), 196-203.

How to cite this article: Koç, M., Dongaz, Ö.İ., Bayar, B. and Bayar, K. (2020). Comparison of Selected Physical and Performance Characteristics In University- Level Male Basketball, Football and Volleyball Players. Int $J$ Disabil Sports Health Sci;3(2):121-127. https://doi.org/10.33438/ijdshs.771545 